NASA Technical Memorandum 101477

ICOMP-89-4

\title{
Phase Development and Its Role on Subharmonic Control
}

Reda R. Mankbadi

Institute for Computational Mechanics in Propulsion

Lewis Research Center

Cleveland, Ohio

Ganesh Raman

Sverdrup Technology, Inc.

NASA Lewis Research Center Group

Cleveland, Ohio

and

Edward J. Rice

National Aeronautics and Space Administration

Lewis Research Center

Cleveland, Ohio

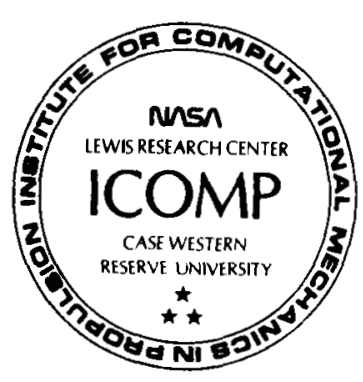


PHASE DEVELOPMENT AND ITS ROLE ON SUBHARMONIC CONTROL

Reda R. Mankbadi, Ganesh Raman, ${ }^{\dagger}$ and Edward J. Rice National Aeronautics and Space Administration

Lewis Research Center

Cleveland, Ohio 44135

\section{Abstract}

The conditions for resonance interaction between two instability waves in an axisymmetric jet were investigated. Considerations of the energy equation of the wave resulting from the interaction indicate that the phase angle between the rave-induced stresses and the wave-induced strains plays a crucial role in the resonance interaction. This fact is demonstrated experimentally by exciting a jet at fundamental and subharmonic frequencies. The phase angle between the waves' stresses and strains was varied by varying the initial phase-difference between the two excitation waves. The subharmonic resonance was found to be highly dependent on this angle. Favorable agreement was found between the phase angles predicted by a nonlinear theory and the measured ones. The theory is used to explain the subharmonic's resonance in terms of the phase-angles.

\section{Introduction}

The recent investigations of Raman, Rice, and Mankbadi (1988) has indicated that improving jet mixing through single frequency excitation has its limitations. Increasing the amplitude of the imposed excitation at a given Strouhal number increases the growth of the momentum thickness of the jet up to a certain level of excitation. Further increase in the excitation level results in a saturation condition beyond which no further increase in jet mixing can be achieved. The experimental observations of Zaman and Hussain (1980) and Ho and Huang (1982) have indicated that a resonance mechanism can occur by which a subharmonic of the fundamental amplifies. The theoretical investigation of Mankbadi (1985) has further indicated that this subharmonic plays a cruclal role in controlling the spreading rate of the jet. Therefore, the present work is concerned with understanding the conditions at which resonance interaction occurs. By properly choosing the initial conditions one may be able to produce resonance interactions and thus achieve further control of jet spreading over that which can be achieved under a single frequency excitation.

The resonance of the subharmonic resulting from its interaction with the fundamental is subject to several parameters such as the Strouhal numbers, the initial levels of both the fundamental and the subharmonic, the turbulence level, and the initial mean velocity profile. Furthermore, for the two-dimensional shear layer Kelly (1967), Zhang, Ho, and Monkewitz (1984) and Monkewitz

*Institute for Computational Mechanics in Propuision (work funded under Space Act Agreement (99066G); on leave from Cairo University. Cairo, Egypt

tsverdrup Technology, Inc., NASA Lewis Research Center Group, Cleveland, OH 44135.
(1988) have indicated that the interaction between the fundamental and the subharmonic is highly dependent on the initial phase-difference between the two waves. Therefore, in a round jet under two-frequency excitation one may expect this phasedifference to play an important role in the subharmonic resonance and in jet mixing.

Cohen and Wygnanski (1987) examined the conditions for resonance interactions between two instability waves. Their analys is is based on assuming a nondivergent mean flow. The two interacting waves are small with respect to the mean flow and the wave resulting from the interaction of the two waves is much smaller in amplitude than any of the two waves. By examining the second order terms in the momentum equation, the condition at wich the particular solution becomes "secular" is to satisfy one of the following conditions:

$$
\omega_{m}=\omega_{\ell}+\omega_{k} ; \quad n_{m}=n_{\ell}+n_{k} ; \quad \alpha_{m}=\alpha_{\ell}+\alpha_{k}
$$

or

$$
\omega_{m}=\omega_{k}-\omega_{l} ; \quad n_{m}=n_{k}-n_{l} ; \quad \alpha_{m}=\alpha_{k}-\alpha_{\ell}
$$

where $\omega$ is the frequency, $n$ is the azimuthal wave number and $\alpha$ is the complex wave number. Subscripts $k$ and $l$ denote the interacting wayes while $m$ denotes the resulting wave. Cohen and Wygnanski (1987) have taken these approximate conditions as the resonance condition.

In this work we re-examine the conditions for resonance interactions for a diverging jet based on the energy equation of the wave resulting from the nonlinear interaction. The conditions on the frequency and azimuthal dependency are found to be the same as those of Cohen and Wygnanski (1987). However, the condition on the wave numbers is replaced by a condition on the phase angle between the waveinduced stresses and the wave-induced strains. To demonstrate experimentally the dependency on this phase angle a round jet is excited at a fundamental and its subharmonic. The phase-difference between the two imposed waves is measured along the jet for different initial phase differences. Measurements of the phase angles are used to verify a nonlinear theory for predicting the phase angle. The latter theory is then used to provide a better understanding of the conditions for resonance interactions.

\section{Energy Considerations of the Wave Interactions}

In the following we examine the waves' energy exchanges. We consider the incompressible flow of a round jet at high Reynolds numbers. The effects of viscosity and the turbulence are ignored in the present analysis.

\section{The Mean Flow-Wave Interactions}

We split the velocity components into mean and pertodic components: 


$$
U_{i}(x, r, \phi, t)=\bar{U}(x, r, \phi)+\bar{u}(x, r, \phi, t)
$$

and

$$
P(x, r, \phi, t)=\bar{P}(x, r, \phi)+\tilde{p}(x, r, \phi, t)
$$

$u_{j}, i=i, 2,3$ are the velocity components in the axial, radial and azimuthal directions, respectively and $P$ is the pressure. $(-)$ denotes timeaveraging while $(\sim)$ denotes a perlodic component. The periodic component is written in terms of Fourier components as:

$$
\begin{aligned}
\tilde{u}_{i}(x, r, \phi, t) & =u_{i, m}(x, r) \exp \left(-i \omega_{m} t+i n_{m} \phi\right) \\
& +u_{j, l}(x, r) \exp \left(-i \omega_{\ell} t+i n_{\ell} \phi\right) \\
& +u_{j, k}(x, r) \exp \left(-\omega_{k} t+i n_{k} \phi\right)+c . C .
\end{aligned}
$$

where C.C. denotes a complex conjugate. $\omega$ is the frequency and $n$ is the azimuthal number. The subscripts $k$ and 2 denote the input waves while the subscript $m$ denotes the wave produced by interaction.

To derive the mean flow energy equation, the decomposition (2.1) is substituted in the full momentum equation. The full momentum equation is then time-averaged and the boundary-layer-type approximations are applied to the mean quantities. upon integrating over $r$, the mean flow energy equation takes the form:

$$
\frac{1}{2} \frac{d}{d x} \int_{0}^{\infty} \bar{u}^{3} r d r=-\int_{0}^{\infty} \bar{u} \bar{v} \frac{\partial \bar{U}}{\partial r} r d r
$$

Thus, the waves can influence the mean flow only through their time-averaged Reynolds shear stress. with the Fourier decomposition (2.2), the timeaveraged wave Reynolds shear stress reduces to:

$$
\begin{aligned}
\tilde{u} \tilde{v}=u_{m} v_{m}^{*}+ & u_{\ell} v_{\ell}^{*}+u_{k} v_{k}^{*}+u_{\ell} v_{m}^{*} \exp \left[-i\left(\omega_{\ell}-\omega_{m}\right) t\right. \\
& \left.+i\left(n_{l}-n_{m}\right) \phi\right]+u_{k} v_{m}^{*} \exp \left[-i\left(\omega_{k}-\omega_{m}\right) t\right. \\
& \left.+i\left(n_{k}-n_{m}\right) \phi\right]+u_{\ell} v_{k}^{*} \exp \left[-i\left(w_{l}-w_{k}\right) t\right. \\
& \left.+i\left(n_{\ell}-n_{k}\right) \phi\right]+C . C .
\end{aligned}
$$

where C.C. or $\left(^{*}\right)$ denote a complex conjugate. The terms appearing in the above expression with dependency on $(\phi)$ are generally zero except in the special case where two of the waves have the same frequency but different azimuthal numbers. For this particular case, these azimuthaly-dependent terms take the form:

$$
u_{Q} v_{k}^{*} \exp \left[i\left(n_{\ell}-n_{k}\right) \phi\right]+C . C .
$$

If integrated over $\phi$ such a term averages to zero indicating that these terms do not contribute to the total mean flow energy in a slice of the jet. These terms only contribute to the azimuthal redistribution of the mean flow energy in the jet. Equation (2.4) also indicates that single excitation with ronaxisymmetric waves cannot disturb the axisymmetry of the mean flow. These conclusions regarding the effect of waves on the mean flow are identical to those of Cohen and Wygnanskt (1987).

\section{Energy of the Wave Resulting from the Interaction}

We derive here the energy equation of the $m$-wave resulting from the interaction of the $k$-wave and the $\ell$-wave. The tine-averaged momentum equation is subtracted from the phase-averaged one. The resulting momentum equation is multiplied by the corresponding velocity component of frequency $\omega$ and the three components of the momentum equations are added and time averaged. The resulting equation is integrated over the radius and the boundary layer-type approximations are applied to the mean quantities. The final form of the integral energy equation of the m-wave is:

$\frac{d}{d x} \int U \overline{Q_{m}^{-}} r d r=\int_{0}^{\infty}-\overline{\bar{u}_{m} \bar{v}} \frac{\partial \bar{U}}{\partial r} r d r$

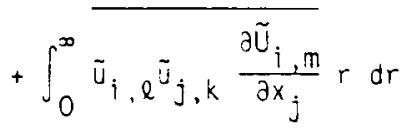

where

$$
\overline{Q_{m}}=0.5\left[\overline{|\bar{u}|^{2}}+|\overline{\tilde{v}}|^{2}+|\bar{w}|^{2}\right]
$$

The first term in the right hand side of the above equation is the energy exchange between the wa'se and the mean flow. The second term in the right hand side is the wave-wave energy exchange. This term will be denoted here by WW.

We view the resonance interaction of the two imposed periodic components as when the wave-wave energy exchange WW is maximum. Therefore, in the following we examine the conditions at which this term is maximum. With the Fourier decomposition given in (2.2) this term is identically zero except if the frequency $\omega$ satisfies one of the following two conditions:

a) $\omega_{m}=\omega_{k}-\omega_{2}$. In this case

$W W=\int u_{i, \ell} u_{j, k}^{*} \frac{\partial \bar{U}_{i, m}}{\partial x_{j}} \exp \left[i\left(n_{\ell}-n_{k}+n_{m}\right) \phi\right]$

$$
x r d r d \phi+C . C \text {. }
$$

b) $\omega_{m}=\omega_{k}+\omega_{\ell}$. In this case

$W W=\int u_{i, \ell}^{*} u^{*}{ }_{j, k} \frac{\overline{\partial U_{i}, m}}{\partial x_{j}} \exp \left[\left(-n_{\ell}-n_{k}+n\right) \phi\right]$

$$
\times r d r d \phi+C . C .
$$

If integrated over $\phi$, WW as given by equation (2.6) integrates to zero except in the special case where the argument of the exponential function is zero. This term thus only redistribute azimuthally the wave energy. For a nonzero wavewave energy in a slice of the jet the azimuthal number of the resulting wave must satisfy one of the following conditions:

$$
n_{m}=n_{k}-n_{\ell}
$$


or,

$$
n_{m}=n_{k}+n_{l}
$$

To examine the conditions on the $x$-dependency for resonance interaction we write:

$$
u(x, r)=\hat{u}(r) F(x)+C . C \text {. }
$$

$\hat{\hat{u}}(r)$ can be obtained as the eigenfunction of the locally parallel linear stability equation for each wave. $F(x)$ is the complex amplitude of each wave which can only be determined from a nonlinear analysis. We can write $F(x)$ as:

$$
F(x)=\sqrt{E(x)} \exp (i \psi(x))+C \cdot C .
$$

$\sqrt{E(x)}$ is the magnitude of the amplitude. With proper normalization of the eigenfunctions, $E(x)$ represents the energy of the wave across the jet. $\psi(x)$ is the phase angle of the wave's energy amolitude.

Let us denote the case where Eqs. (2.6a) and (2.7a) are satisfied as case I and the case where Eqs. (2.6b) dnd (2.7b) satisfied as case II. For both cases the wave-wave energy exchange can be written as:

$$
W W=\sqrt{E_{k}(x) E_{m}(x) E_{l}(x)}\left|I_{W}\right| \cos (\gamma)
$$

where

$$
\begin{aligned}
I_{W} \int_{0}^{\infty} & =\hat{u}_{i, \ell} \hat{u}_{j, k}^{*} \frac{\partial \hat{u}_{i, m}}{\partial x_{j}} r d r \quad \text { for case I } \\
& =\int_{0}^{\infty} \hat{u}_{i, \ell}^{*} \hat{u}_{j, k}^{*} \frac{\partial \hat{u}_{i, m}}{\partial x_{j}} r d r \quad \text { for case II }
\end{aligned}
$$

and

$$
\begin{aligned}
\gamma & =\psi_{m}+\psi_{l}-\psi_{k}+\sigma & & \text { for case I } \\
& =\psi_{m}-\psi_{l}-\psi_{k}+\sigma & & \text { for case II }
\end{aligned}
$$

$\sigma$ is the phase angle of $I_{W}$. We examine now the $x$-dependency of $W W$ as given by Eq. (2.8) $I_{W}$ is obtained from the local linear stability theory for a given set of strouhal numbers. For the case of fundamental subharmonic interaction this function is shown in Fig. 1. The axial dependency is replaced here by the momentum thickness which is a function of the axial location $(x)$. The Strouhal number shown on the figure is that of the fundamental. For a given Strouhal number, the wave-wave interaction is a function of $I_{W}$, its phase $\sigma$, and the energy of each wave (Eq. 2.8). The figure shows that the general trend of $I_{W}$ is to decrease with increasing the momentum thickness. Thus, the smaller the initial momentum thickness, the stronger the wave-wave interaction can be. As Eq. (2.8) indicates, the wave-wave interaction is proportional to the product of the energy levels of the three waves. These levels are dependent on their initial levels, among other parameters. Therefore, small amplitude excitation may not produce resonance interactions even if all other conditions are met. Finally, we consider the dependency of the wave-wave interaction on the angle $(\gamma)$. All functions appearing in Eq. (2.8), except $\cos (y)$, varies monotonically with $x$. In general $\cos (y)$ has an oscillatory nature that can cancel the wave-wave interaction if integrated over $x$. To achleve resonance interactions, the argument of the cosine should ideally be zero. This condition can be written as:

$$
\begin{array}{ll}
\psi_{m}=\psi_{k}-\psi_{l}-\sigma & \text { for case I } \\
\psi_{m}=\psi_{k}+\psi_{l}-\sigma & \text { for case II }
\end{array}
$$

To examine the physical meaning of this condition, we can write WW as:

$W W=\int_{0}^{\infty} \tilde{u}_{i, \ell} \tilde{u}_{j, k} \frac{\partial \tilde{u}_{\tilde{i}}, m}{\partial x_{j}} r d r \approx \bar{R} \cdot \bar{s}$

$$
=|R||S| \cos (y) \quad(2.10)
$$

Where $\vec{R}$ and $\vec{S}$ are vectors representing the radially-averaged stresses and strains, respectively. The condition stated in Eq. (2.9) is thus equivalent to requiring the wave-induced stresses and the wave-induced strains to be in-phase. Equation (2.10) indicates that this angle plays an essential role in the subharmonic resonance. If $\gamma=0^{\circ}$, we obtain maximum positive energy transfer to the output wave resulting in the resonance condition. If $Y=180^{\circ}$, we obtain maximum energy drain from the output wave resulting in suppressing the output wave. This latter situation is observed experimentally as the "vortex-shredding" (e.g.. Monkewitz, 1987). If $y=90^{\circ}, w w=0$ which indicates negligible interactions and that the m-wave develops as if the other two waves are almost not present.

The conditions for resonance interactions can thus be summarized as follows:

$$
\left.\begin{array}{c}
\omega_{m}=\omega_{k}-\omega_{l}, \quad n_{m}=n_{k}-n_{l}, \\
\psi_{m}=\psi_{k}-\psi_{\ell}-\sigma \\
\text { or } \\
\omega_{m}=\omega_{k}+\omega_{l}, \quad n_{m}=n_{l}+n_{k}, \\
\psi_{m}=\psi_{k}+\psi_{l}-\sigma
\end{array}\right\}
$$

The conditions on the frequency and on the azimuthal number are identical to those given by conen and Wygnanski (1987). However, the condition on the phase angle is different from that on the wave numbers given by Cohen and Wygnanski. Unlike Conen and Wygnanski's (1987) analysis, the present analysis does not require the flow to be nondiverging or that the waves to be weakly nonlinear. To examine the relations between the present result and that of Cohen and Wygnanski, we consider the axisymmetric case of fundamental-subharmonic interaction. The present condition on the phase angle reduces to the form:

$$
Y=2 \psi_{S}-\psi_{f}+\sigma=0
$$

where the subscript $f$ denotes the fundamental of frequency $2 \omega$ and $s$ denotes the subharmonic of frequency $\omega$. If we take the phase angle to be 
approximately given by the linear stability theory. Ea. (2.12) can be written as:

$$
2 \alpha_{r s} \times-\alpha_{r} f \times \psi_{0}+\sigma=0
$$

where $\alpha_{r}$ is the real part of the complex wave number obtained as the eigenvalue of the linear stability equation. $\Psi_{0}$ is the phase angle by which the fundamental initially leads the subharmonic. The corresponding condition given by cohen and Wygnanski is:

$$
2 \alpha_{r s}-\alpha_{r} f=0
$$

It can be shown that the above condition is equivalent to requiring equal phase velocities. Conen and Wygnanski's condition (Eq. (2.14a)) and the present condition (Eq. (2.13)) becomes identical under the following conditions: (1) the linear stability theory provides a valid approximation to the phase angles of the waves, (2) the initial. phase difference between the two waves is ignored, and (3) the phase angle of $I_{W}, \sigma$, is set identical to zero.

The ability of the linear stability theory to represent the axial variation of the phase angles will be examined later on in this work. The effect of initial phase difference on the subharmonic resonance cannot be ignored. Monkewitz's (1987) observations for a two-dimensional shear layer under two-frequency excitation clearly indicates that the initial phase-difference controls the subharmonic resonance. The variation of the angle ( $\sigma$ ) along the jet was shown in Fig. 1. The figure clearly indicates that this angle varies considerably along the jet and cannot be set equal to zero.

Cohen and Wygnanski (1987) attempted to demonstrate experimentally the condition given by Eq. (2.14). A jet was excited at $288 \mathrm{~Hz}$ and the subharmonic at $144 \mathrm{~Hz}$ was found to amplify. When the excitation frequency was changed to $144 \mathrm{~Hz}$ the corresponding subharmonic at $72 \mathrm{~Hz}$ was not amplified. Since the $288 \mathrm{~Hz}$ wave and the $144 \mathrm{~Hz}$ wave have the same phase speed while the 144 and $72 \mathrm{~Hz}$ do not, they concluded that this supports the condition given by Eq. (2.14). However, the amplification of the subharmonic at $144 \mathrm{~Hz}$ can be explained from the linear theory since in their experiment the $144 \mathrm{~Hz}$ corresponded to the most amplified wave while the $72 \mathrm{~Hz}$ corresponded to a weakly amplified wave. This fact, and not the equality of the phase velocities, can therefore explain the subharmonic resonance when the jet was excited at $288 \mathrm{~Hz}$.

Actually Cohen and Wygnanski's (1987) data provide an example which clearly violates the condition of Eq. (2.14) which for the general case takes the form

$$
\alpha_{m}=\alpha_{k} \pm \alpha_{\ell}
$$

In their fig. $7(c)$ a jet was excited at two frequencies 288 and $192 \mathrm{~Hz}$. A wave corresponding to the difference $96 \mathrm{~Hz}$ was amplified. If one substitutes the wave numbers $\left(\alpha_{r}\right)$ as obtained from the inear stability theory corresponding to these frequencies, Ea. (2.14b) is about 50 percent in error. This suggests that their condition on the wave numbers (Eq. 2.14b), is only an approximate one.
In the following we attempt to demonstrate experimentally that the phase-angle, not the phase velocity, controls the subharmonic resonance.

\section{Experimental Apparatus and Procedure}

The jet facility contains a 76-cm diameter plenum chamber, a two-stage contraction followed by an 8.8-cm diameter nozzle. A $41-\mathrm{cm}$ diameter section located between the two contracting sections contains the excitation system, which consists of two Ling drivers. These Ling electropneumatic drivers were used to produce the high excitation amplitude required for the present set of tests. These drivers have an electrodynamically operated pneumatic valve capable of reproducing sine, random or any complex wave form and can generate up to $4000 \mathrm{~W}$ of acoustic power per oriver within the operating range. A polynominal wave form synthesizer is used to produce the two frequencies at the desired initial phasedifference. The wave form synthesizer generates wave shapes from user supplied mathematical expressions.

The phase-difference, $\beta$, in the experiment is defined with respect to the fundamental, i.e..

$$
\begin{gathered}
\tilde{u}_{f} \sim \sin \left(\omega_{f} t+\beta\right) \\
\tilde{u}_{S} \sim \sin \left(\omega_{s} t\right)
\end{gathered}
$$

\section{Experimental Results}

Since the purpose here is to examine the effect of varying the initial phase-difference, it is necessary to show that the other initial parameters are kept constant. The exit profiles of the mean velocity and the total fluctuations are shown in Fig. 2 for a Strouhal number pair of 0.2 and 0.4 . Both the unexcited and the excited cases at $\beta_{0}=90^{\circ}$ and $270^{\circ}$ are shown for a Strouha $i$ number pair of 0.2 and 0.4 . The figure shows that the initial mean velocity profile for both the excited and the unexcited cases are the same. The exit momentum thickness is $0.015 R$, where $R$ is the nozzle's radius. For the unexcited case the turoulence intensity at the jet centerline and boundary layer are 2 and 8 percent, respectively. Since these total fiuctuations are composed of the background turbulence as well as the imposed waves, the total fluctuations for the excited case increases over that of the unexcited case due to the imposed waves. For the excited case the figure indicates that the levels of the imposed fluctuations are the same for the $\beta_{O}=90^{\circ}$ and the $\beta_{O}=270^{\circ}$ cases.

Dependency of the subharmonic's amolification on the initial phase-difference. The phase-averaging technique is used to educt the fundamental and the subharmonic components along the centerline of the jet. The fundamental and subharmonic axial velocities along the jet centerline are shown in Fig. 3 for several initial phase-differences. The fundamental is not sensitive to the initial phasedifference but the amplification of the subharmonic is quite sensitive to the initial phase-difference. This is demonstrated further in Fig. 4 which is a polar plot of the subharmonic's peak against the initial phase angle. The figure shows that the subharmonic is maximum at an initial phase-difference 
of $270^{\circ}$ and is minimum at $90^{\circ}$. The initial phasedifference can thus result in either a subharmonic's resonance or a subharmonic's suppression.

One of the findings of the energy considerations in the previous section is that the wave-wave energy exchange mechanism is proportional to $E_{5} \vee E_{f}$, (Eq. (2.8)). Thus, this mechanism is important only when the product $E_{s} \sqrt{E_{f}}$ exceeds a certain level. This fact is demonstrated in Fig. 5 by increasing the fundamental level from 1 to 7 percent. The subharmonic-fundamental initial ratio is kept at $1: 15$. Thus, in Fig. 5 both the subharmonic's level and the fundamental's level are increased and hence $E_{S} \sqrt{E_{f}}$ is aiso increased. The initial phase-difference is kept at $270^{\circ}$. Figure 7 clearly indicates that the subharmonic's ampification increases with increasing the product of the energies $E_{s} \sqrt{E_{f}}$, in accordance with Eq. (2.8))

Development of the phase-difference. In the present section we examine the development of the phase-difference along the jet and its dependency on the initial conditions.

The phase-difference minus the initial phasedifference, $\beta-\beta_{0}$, is shown in Fig. 6 as a continuous function of $x / D$. The strouhal numbers are 0.2 and 0.4 , Mach number $0.45, \tilde{u}_{f}=7$ percent. $\tilde{u}_{s}=0.5$ percent. The figure shows that the development of the phase-difference along the jet is highly infiuenced by its initial value. This indicates that the development of the phase-difference is a nonlinear process. In a linear theory, the phase angles are given by the local mean flow parameters irrespective of the initial conditions. The momentum thickness along the jet for $\beta_{0}=90^{\circ}$ and $270^{\circ}$ are shown in Fig. 7. The two values of Bo correspond to the maximum and minimum subharmonic amplification. The figure shows that the local momentum thickness is almost the same for both values of $\beta_{0}$. Since in a linear theory, the phase angle is determined by the local mean flow parameters (irrespective of the initial conditions). The fact that the momentum thickness is almost the same for both initial phase-differences indicates that a linear theory cannot be used to predict the development of the phase-difference along the jet.

To examine further the nonlinearity of the phase angles, the initial level of the fundamental is varied from 1 to 7 percent of the jet exit velocity. The ratio of the subharmonic to the fundamental at the exit is kept almost constant at $1: 15$. Figure 8 shows that the initial level of the fundamental has a pronounced effect on the development of the phase-difference particularly for $x / 0>2$.

Prediction of the nonlinear development of the phase-difference

The nonlinear development of the phase angle was considered by Mankbadi (1986) for a laminar jet. The wave components are taken in the form: $\tilde{u}_{f}=F_{f}(x) \tilde{u}_{f}(r) \exp \left(-i \omega_{f} t+\psi_{0}\right)+C . C$.

$\tilde{u}_{s}=F_{s}(x) \hat{u}_{s}(r) \exp \left(-i \omega_{s} t\right)+C \cdot C$.

$\hat{u}_{f}$ and $\hat{u}_{j}$ are the eigenfunctions obtained from the solution of the linearized inviscid momentum equations for each wave component at the corresponding frequency. $F f(x)$ and $F_{S}(x)$ are the complex amplitudes of the fundamental and subharmonic, respectively, which can be written as:

$$
\begin{aligned}
& F_{f}(x)=\sqrt{E_{f}(x)} e^{i \psi f} \\
& F_{S}(x)=\sqrt{E_{S}(x)} e^{i \psi s}
\end{aligned}
$$

Comparing Eq. (4.1) to the definition of the phaseangle in the experiment, ( $E q .3 .1$ ), the relation between $\beta$ and $\psi$ is given by:

$$
\beta=-\left[\psi_{f}-\psi_{s}+\left(\Delta_{f}-\Delta_{s}\right)+\psi_{0}\right] \text {. }
$$

which gives $\beta_{0}=-\left(\Delta_{f}-\Delta_{S}+\psi_{0}\right)$, where $\Delta$ denotes the phase angle of the eigenfunction $\dot{u}$.

The eigen functions are normalized such that $\varepsilon_{f}$ and $E_{s}$ represent the energy of each wave integrated across the jet. The equation governing the nonlinear development of the phase angle of the subharmonic is obtained as follows. The nonlinearized $x$-momentum equation of the total periodic velocity component is multiplied by $F_{s}^{*} \exp (i w t)$, where (*) denotes a complex conjugate. The $y$-momentum equation is similarly multiplied by $F_{s}(x) v_{s}^{*} \exp (i \omega t)$ and the $x$ - and $y$-equations are added and time-averaged. This equation is simplified by applying the usuai boundary-layer-type approximations to the mean quantities. The resulting equation is then subtracted from its complex conjugate. A similar procedure is used to obtain an equation for the fundamental's phase angle. The final equations are given in Mankbadi (1986) as:

Fundamental:

$$
\begin{aligned}
I_{c f} \frac{d \psi_{f}}{d x}=\pi S_{f}+G\left(S_{f}, \theta\right) \\
\quad-\frac{E_{S}}{\sqrt{E_{f}}}\left|I_{W}\right| \sin \left(2 \psi_{S}-\psi_{f}-\psi_{o}+\sigma\right)
\end{aligned}
$$

Subharmonic:

$$
\begin{aligned}
& I_{C S} \frac{d \psi_{S}}{d x}=\pi S_{S}+G\left(S_{S}, \theta\right) \\
& \quad+\sqrt{E_{f}}\left|I_{W}\right| \sin \left(2 \psi_{S}-\psi_{f}-\psi_{O}+\sigma\right)
\end{aligned}
$$

where:

$$
\begin{aligned}
& G=I_{m}\left[\int_{0}^{\infty} \hat{u v^{*}} \frac{\partial U}{\partial r} r d r\right], \\
& \sigma=\tan ^{-1}\left[I_{m}\left(I_{W}\right) / R_{e}\left(I_{W}\right)\right]
\end{aligned}
$$

The solution of $\mathrm{Eq}$. (4.3) is subject to the ini$t i a l$ values of the phase angles.

To compare the results of this nonlinear theory with the observations, the energies of the two waves $E_{f}(x)$ and $E_{s}(x)$ are estimated from the measurements of the fundamental and subharmonic 
relcc:ties for a given set of initial conditions and are used as specified functions in solving Eq. (4.3)

The solution of Eq. (4.3) for initial phasedifferences of $90^{\circ}$ and $270^{\circ}$ is compared with the corresponding experimental data in Fig. 9. Since this is a nonlinear theory. the development of the phase-difference depends on the magnitude of each wave component and hence on the inftial conditions which are taken from the experiment. The figure shows that the prediction of the present nonlinear theory compares favorably well with the data. The theory indicates that the phase-difference increases along the jet and is larger at $\beta=90^{\circ}$ than the corresponding phase-difference at $B_{0}=270^{\circ}$. The same features are exhibited in the measured data. The prediction of the phasedifference is aiso compared with the measured data in Fig. 10 for a Strouhal number pair of 0.3 and 0.6 . The figure shows reasonably well comparison between theory and observations. However, the theory does not quite match the experiment. As $x$ increases the turbulence intensity increases. The present theory ignores the background turbulence which accounts for the deviation between the theory and the observations.

\section{Conclusions}

The corditions for subharmonic's resonance in a two-frequency excitation of a round jet were investigated. The data indicate that the subharmonic resonance is strongly dependent on the initial onase-difference between the two imposed vaves. Subharmonic's resonance is viewed in terms of the energy transfer from the fundamental to the subharmonic. This energy transfer is optimum when the subnarmonic-induced stresses are in-phase with the fundamertal induced strain. The initial phasedifference between the two imposed waves controls the stress-strain angle and determines whether the fundamental-subharmonic interactions would result in ennancing or suppressing the growth of the subharmonic.

\section{References}

1. Cohen, J.; and Wygnanski, I.: The Evolution of Instabilities in the Axisymmetric Jet. Part 2. The Flow Resulting from the Interaction Betiveen Two Waves. J. Fluid Mech., vol. 176, Mar. 1987, pp. 221-235.
2. Ho, C.M. : and Huang, L.S.: Subharmonics and Vortex Merging in Mixing Layers. J. Fluid Mech., vol. 119, June, 1982, pp. 443-473.

3. Kelly, R.E.: On the Stability of an Inviscid Shear Layer Which is Periodic in Space and Time. J. Fluld Mech., vol. 27, pt. 4, Mar. 20, 1967, pp. 657-689.

4. Mankbadi, R.R.: The Mechanism of Mixing Enhancement and Suppression in a Circular Jet Under Excitation Conditions. Phys. Fluids, vol. 28, no. 7, July, 1985, pp. 2062-2074.

5. Mankbadi, R.R.: The Effect of Phase-Difference on the Spreading Rate of a Jet. AIAA J., vol. 24 , no. 12, Dec. 1986 , pp. $1941-1948$.

6. Michalke, A.: Instabilitat Eines Kompressiblen Runden Freistrahls Unter Berucksichtigung des Einfluesses der Strahlgrenzschichtdicke. Z. Flugwiss, vol. 19, no. 8/9, Aug.-Sept. 1971, pp. 319-328.

7. Monkewitz, P.A.: Subharmonic Resonance, Pairing and Shredding in the Mixing Layer. J. Fluid Mech., vol. 188, Mar. 1988, po. 223-252.

8. Raman, G.; Rice, E.J.; and Mankbadi, R.R.: Saturation and the Limit of Jet Mixing Enhancement by Single Frequency Plane Wave Excitation: Experiment and Theory. AIAA Paper 88-3613, July 1988 (NASA TM-100882).

9. Zaman, K.B.M.Q. : and Hussain, A.K.M.F.: Vortex Pairing in a Circular Jet Under Controlled Excitation. Part I. General Jet Response. J. Fluid Mech., vol. 101, pt. 3, Dec. 11, 1980, pp. $449-491$.

10. Zhang, Y.Q.; and Ho, C.M.; and Monkewitz, P.: The Mixing Layer Forced by Fundamental and Subharmonic. Laminar-Turbulent Transition, V.V. Kozlov, ed., Springer-Verlag. 1984. pp. $385-396$ 


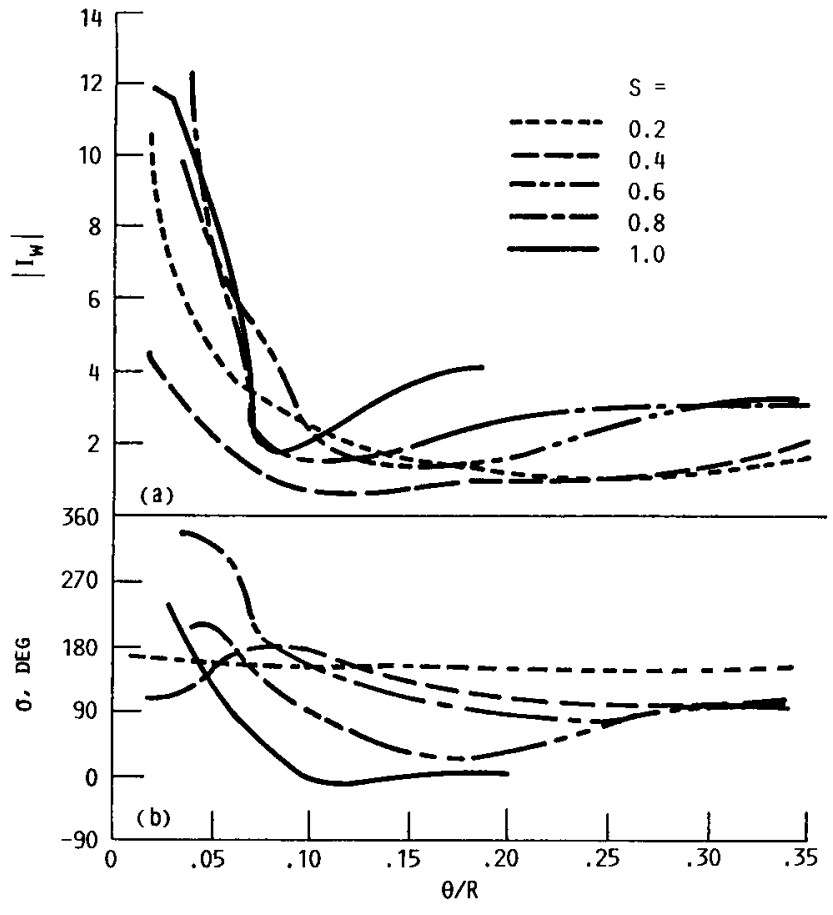

FIGURE 1. - THE WAVE-WAVE INTEGRAL I AT DIFFERENT STROUHAL NUMBERS OF THE FUNDAMENTAL. (a) MAGNITUDE. (b) PHASE,

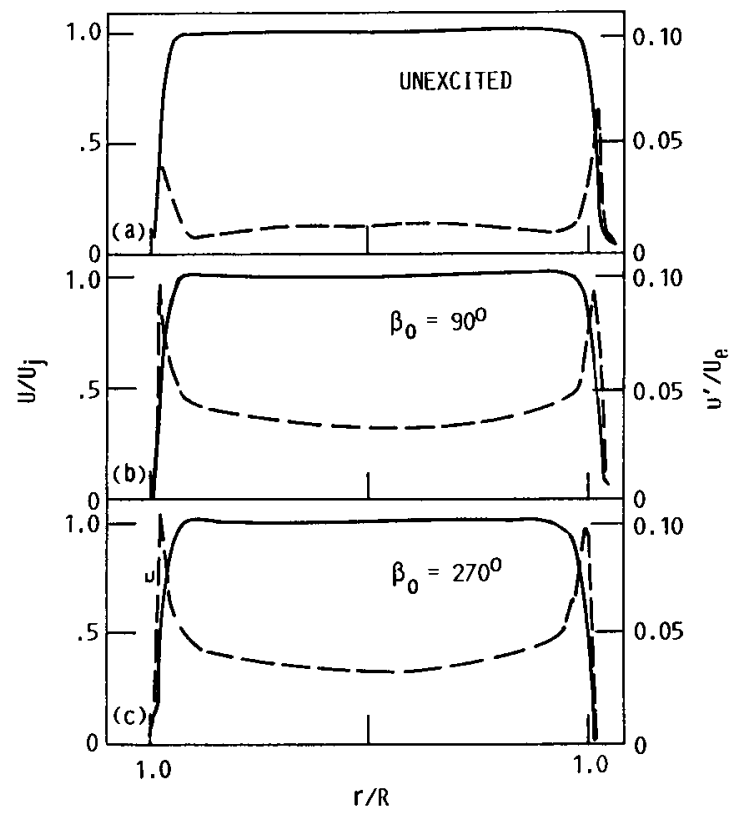

FIGURE 2. - THE EXIT PROFILES OF THE MEAN VELOCITY AND THE TOTAL FLUCTUATIONS. (a) UNEXCITED. (b) EXCITED AT $\beta_{0}=90^{\circ}$. (c) EXCITED AT $\beta_{0}=270^{\circ}$.

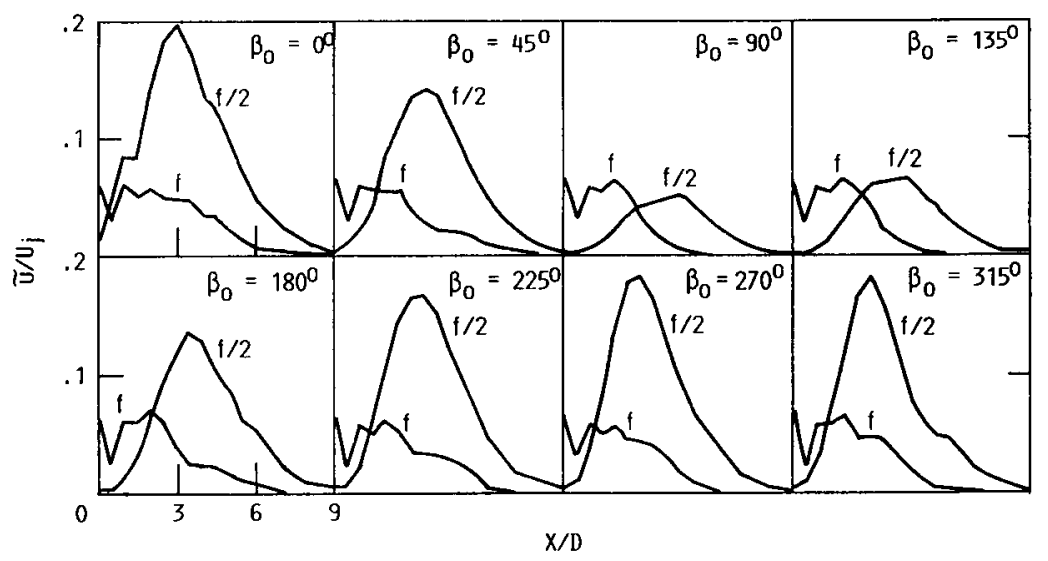

FIGURE 3. - PHASE-AVERAGED FUNDAMENTAL'S AND SUBHARMONIC'S AXIAL VELOCITY COMPONENTS AT SEVERAL INITIAL PHASE-DIFFERENCES. 


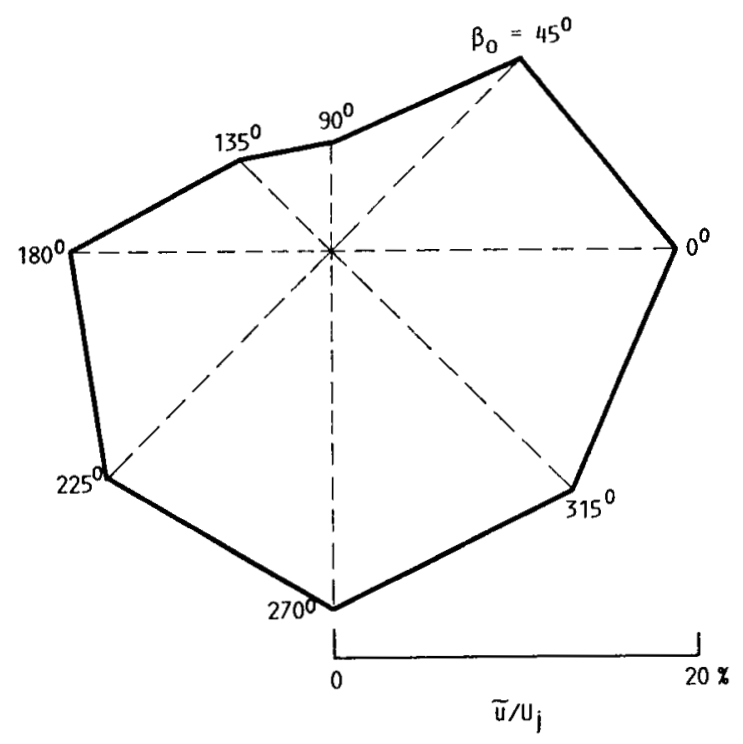

FIGURE 4. - POLAR PLOT OF THE MAXIMUM OF THE SUBHARMONIC AS A FUNCTION OF THE INITIAL PHASE-DIFFERENCE.

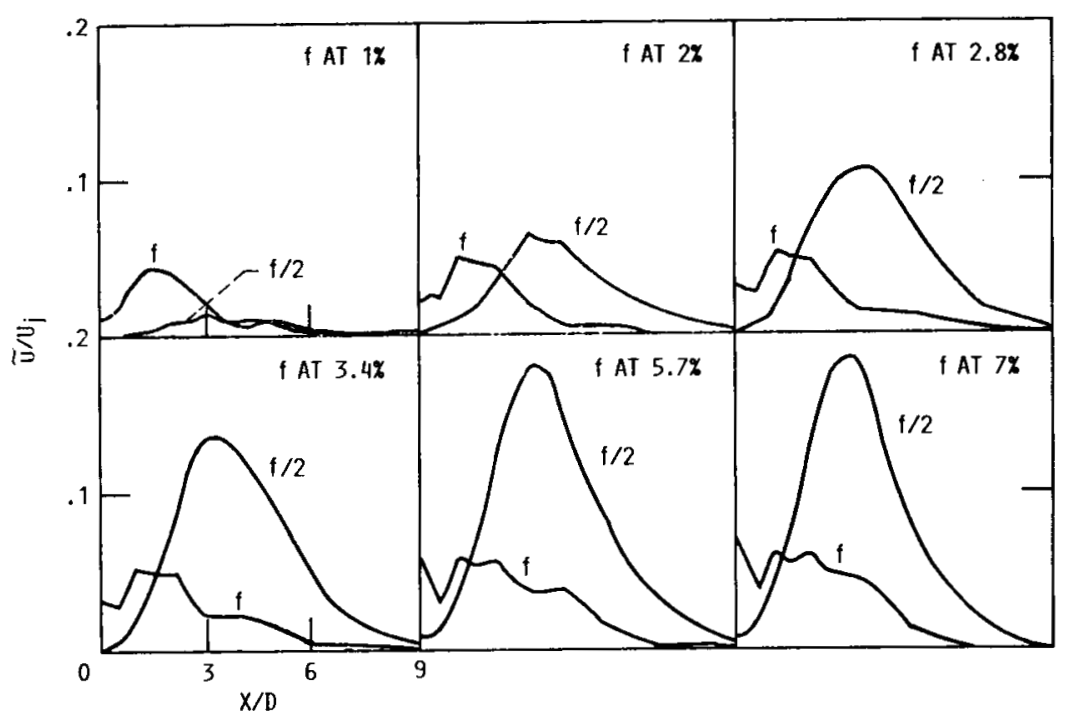

FIGURE 5. - DEPENDENCY OF THE SUBHARMONIC'S AMPLIFICATION ON THE INITIAL LEVELS. MACH NUMBER $=0.45$ AND STROUHAL NUMBER PAIR $=0.2$ AND $0.4 \mathrm{AT}$ $B_{0}=270^{\circ}$. 


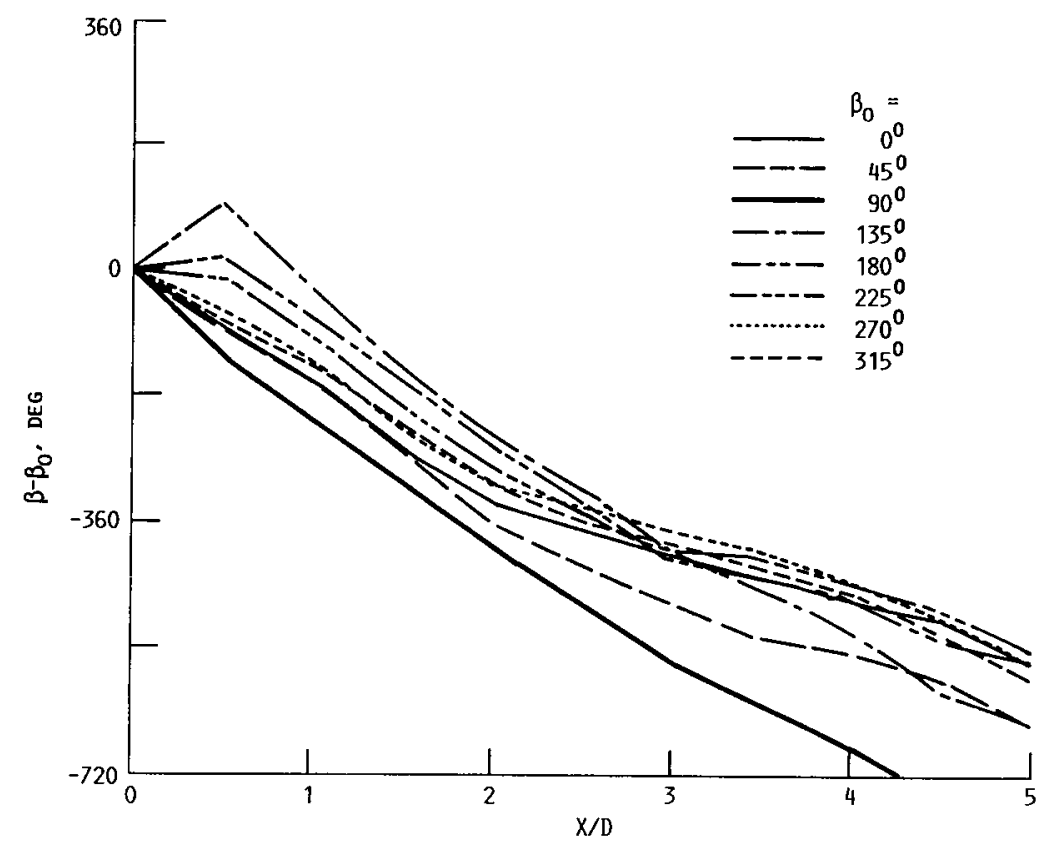

FIGURE 6. - MEASURED PHASE-DIFFERENCE BETWEEN THE TWO WAVES FOR SEVERAL INITIAL PHASE-DIFFERENCES.

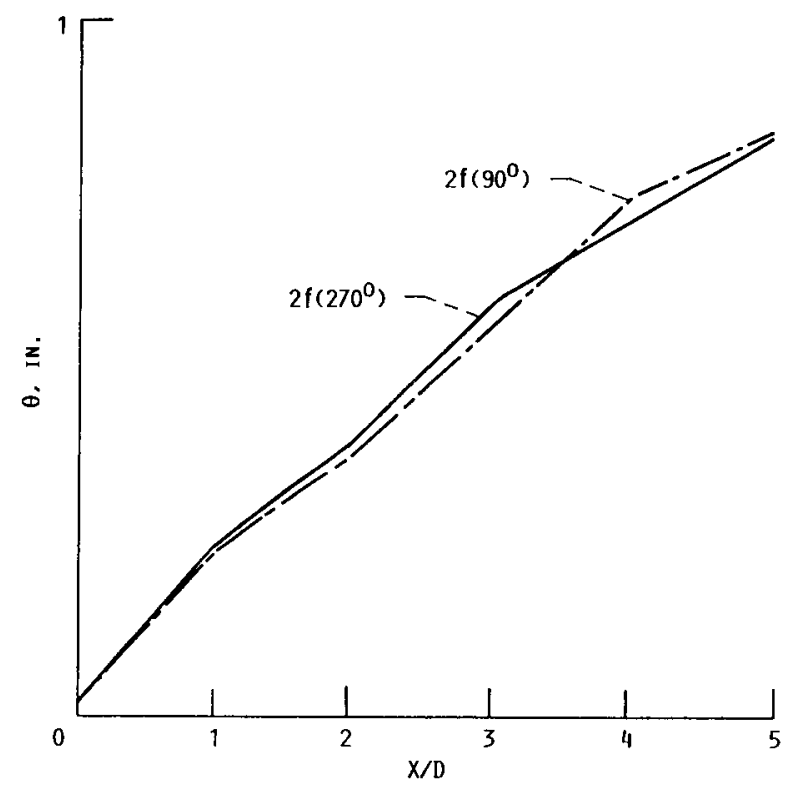

FIGURE 7. - THE LOCAL MOMENTUM THICKMESS UNDER TWOFREQUENCY EXCITATIONS AT $\beta_{O}=90^{\circ}$ AND $\beta_{O}=270^{\circ}$. 


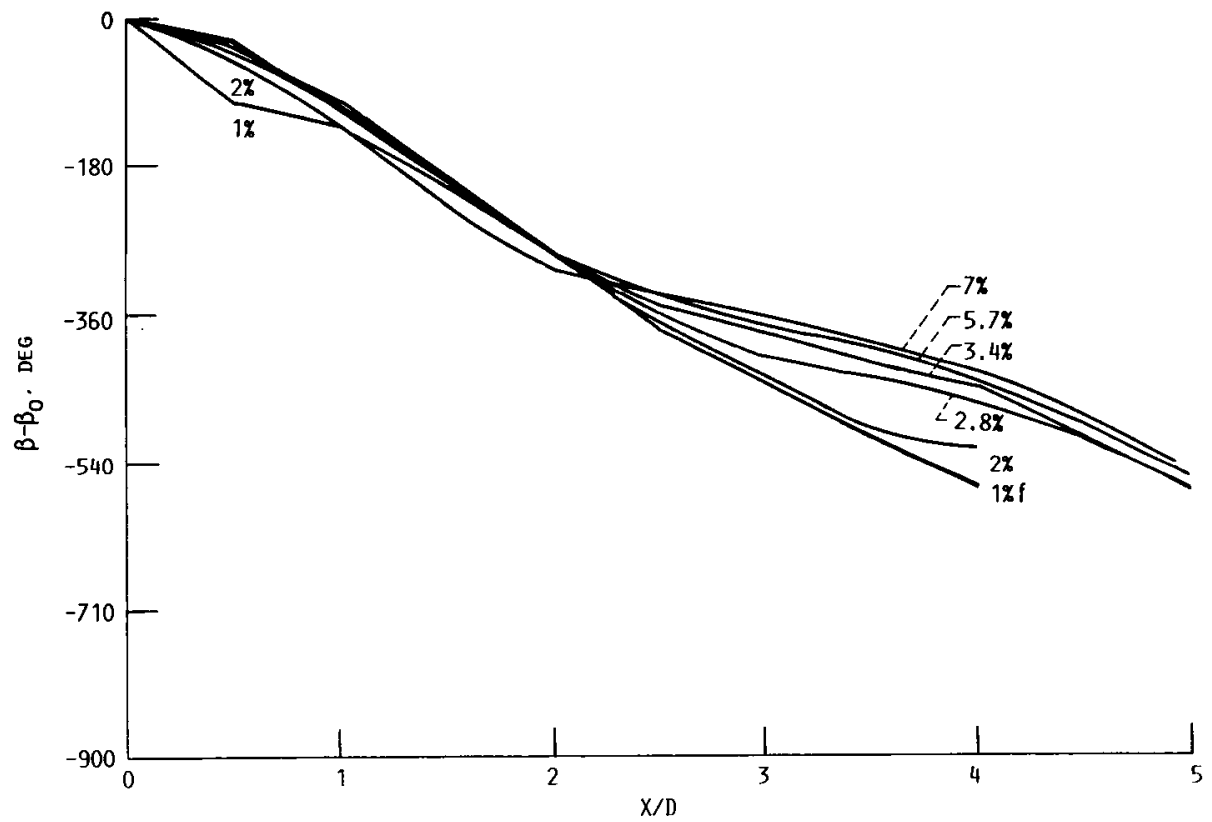

FIGURE 8. - EFFECT OF THE INITIAL LEVEL OF THE FUNDAMENTAL ON THE DEVELOPMENT OF THE PHASE-DIFFERENCE ALONG THE JET. THE SUBHARMONIC'S INITIAL LEVEL IS 1/15 OF THAT OF THE FUNDAMENTAL. 

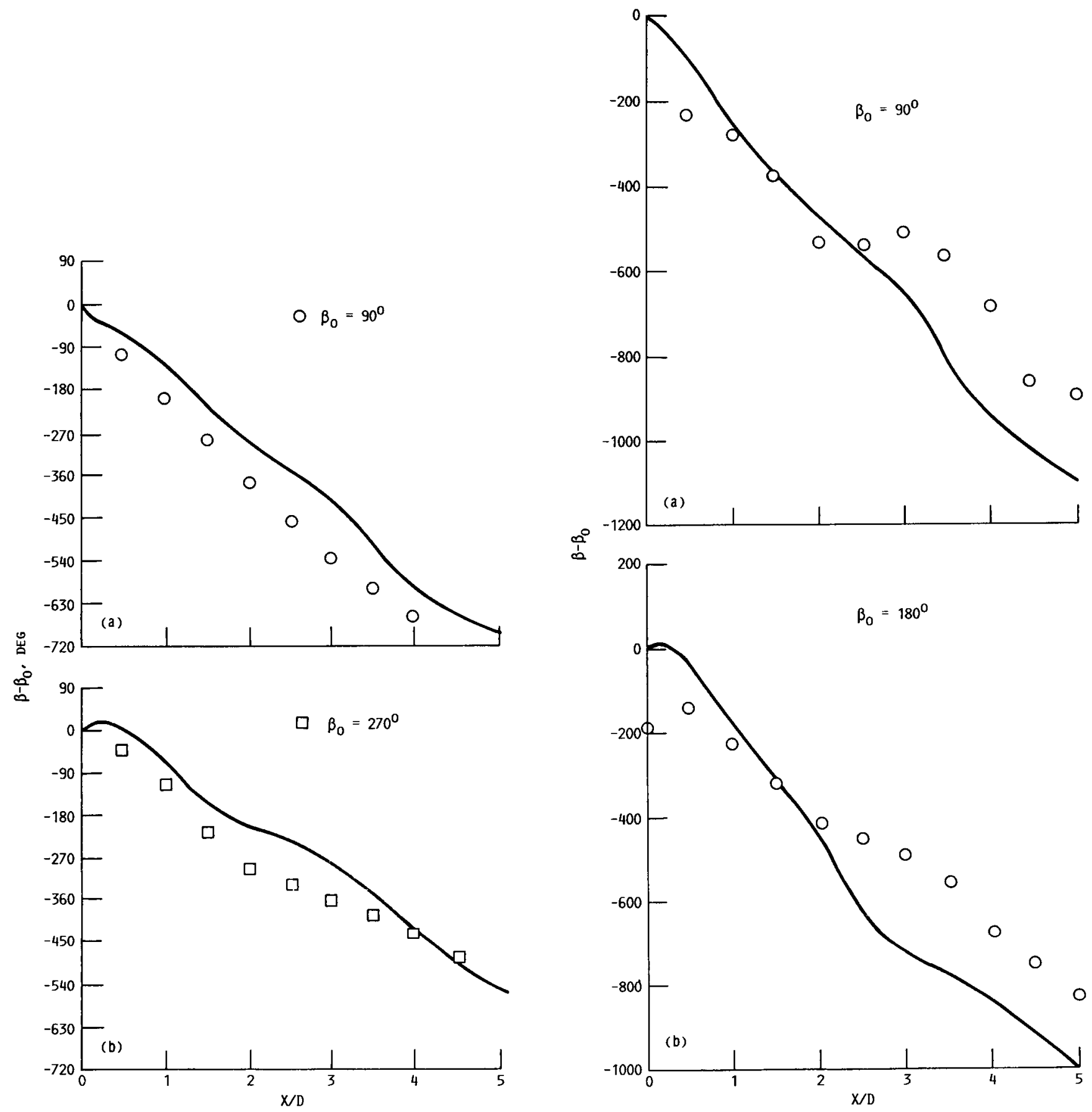

FIGURE 9 - COMPARISON BETWEEN PREDICTED AND MEASURED PHASE-DIFFERENCE FOR $\beta_{0}=90^{\circ}$ AND $\beta_{0}=270^{\circ}$. OTHER INITIAL CONDITIONS ARE THE SAME AS IN FIGURE 4. (a) $\beta_{0}=90^{\circ}$. (b) $\beta_{0}=270^{\circ}$.

FIGURE 10. - COMPARISON BETWEEN PREDICTED AND MEASURED PHASE-DIFFERENCES ALONG THE JET FOR A STOUHAL NUMBER PAIR $=0.3$ AND 0.6. (a) $\beta_{0}=90^{\circ}$. (b) $\beta_{0}=180^{\circ}$. 


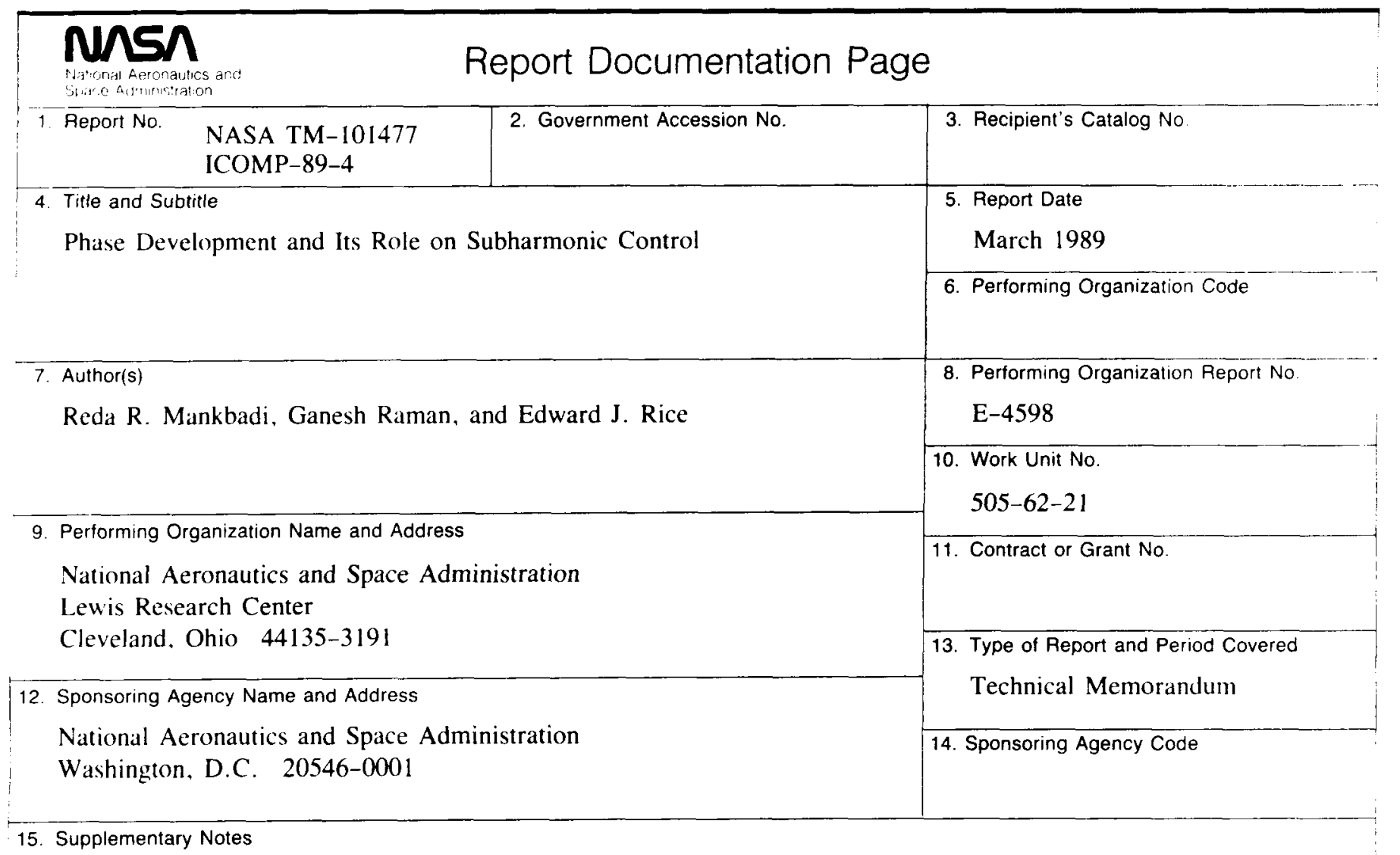

Reda R. Mankbadi, Institute for Computational Mechanics in Propulsion, Lewis Research Center (work funded under Space Act Agreement C99066G); on leave from Cairo University, Cairo, Egypt. Ganesh Raman. Sverdrup Technology, Inc., NASA Lewis Research Center Group, Cleveland, Ohio 44135. Edward J. Rice, NASA Lewis Research Center.

16. Abstract

The conditions for resonance interaction between two instability waves in an axisymmetric jet were investigated. Considerations of the energy equation of the wave resulting from the interaction indicate that the phase angle between the wave-induced stresses and the wave-induced strains plays a crucial role in the resonance interaction. This fact is demonstrated experimentally by exciting a jet at fundamental and subharmonic frequencies. The phase angle between the waves stresses and strains was varied by varying the initial phase-difference between the two excitation waves. The subharmonic resonance was found to be highly dependent on this angle. Favorable agreement was found between the phase angles predicted by a nonlinear theory and the measured ones. The theory is used to explain the subharmonic's resonance in terms of the phase-angles.

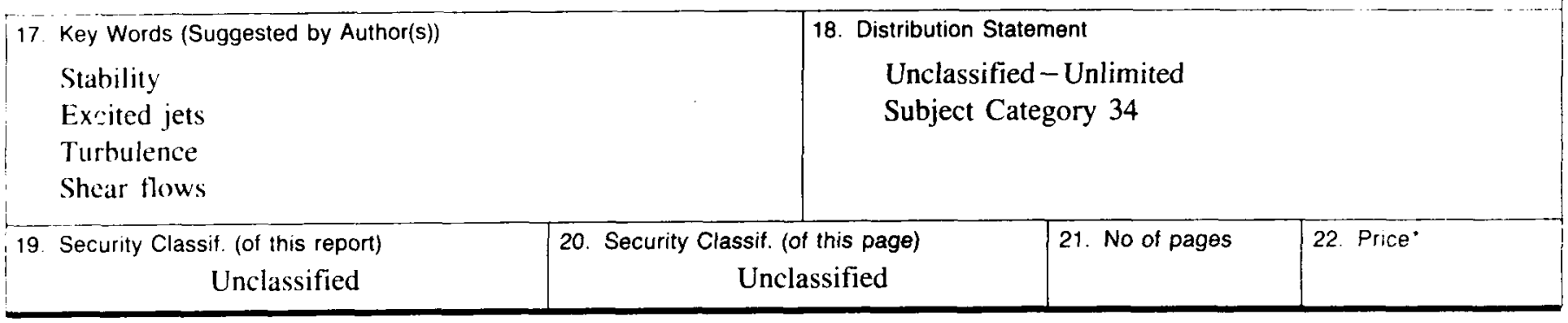

